\title{
LETTER
}

Chronic myeloproliferative neoplasms

\section{Safety and efficacy of the maximum tolerated dose of givinostat in polycythemia vera: a two-part Phase Ib/ll study}

\author{
Alessandro Rambaldi $\mathbb{D i D}^{1} \cdot$ Alessandra lurlo $\mathbb{D}^{2} \cdot$ Alessandro M. Vannucchi $^{3} \cdot$ Richard Noble ${ }^{4}$ Nikolas von Bubnoff ${ }^{5,6}$. \\ Attilio Guarini ${ }^{7} \cdot$ Bruno Martino $^{8} \cdot$ Antonio Pezzutto $^{9}{ }^{9} \cdot$ Giuseppe Carli $^{10}$ - Marianna De Muro ${ }^{11} \cdot$ Stefania Luciani $^{12}$. \\ Mary Frances McMullin ${ }^{13}{ }^{13} \cdot$ Nathalie Cambier $^{14} \cdot$ Jean-Pierre Marolleau $^{15} \cdot$ Ruben A. Mesa ${ }^{16} \cdot$ Raoul Tibes $^{17} \cdot$ \\ Alessandro Pancrazzi ${ }^{3}$. Francesca Gesullo ${ }^{3}$ Paolo Bettica ${ }^{18} \cdot$ Sara Manzoni $^{18} \cdot$ Silvia Di Tollo $^{18}$
}

Received: 15 October 2019 / Revised: 6 December 2019 / Accepted: 29 January 2020 / Published online: 11 February 2020

(c) The Author(s) 2020. This article is published with open access

\section{To the Editor:}

Polycythemia vera (PV) is a chronic myeloproliferative neoplasm (cMPN) characterized by stem cell-derived clonal myeloproliferation resulting in panmyelosis with persistently raised hematocrit, increased risk of thrombotic complications, and predisposition to evolve to myelofibrosis or leukemia [1]. Therapy is currently based on phlebotomy to normalize hematocrit, and aspirin. Hydroxyurea is used as first line when cytoreduction is necessary [1], although toxicity can result in inadequate disease management [2].

Supplementary information The online version of this article (https:// doi.org/10.1038/s41375-020-0735-y) contains supplementary material, which is available to authorized users.

Alessandro Rambaldi

alessandro.rambaldi@unimi.it

1 Department of Oncology and Hematology, University of Milan and ASST Papa Giovanni XXIII, Bergamo, Italy

2 Hematology Division, Fondazione IRCCS Ca' Granda Ospedale Maggiore Policlinico, Milan, Italy

3 Department of Experimental and Clinical Medicine, University of Florence, Azienda Ospedaliera-Universitaria Careggi, Florence, Italy

4 Department of Hematology, Royal Cornwall Hospital, Truro, UK

5 Department of Hematology, Oncology and Stem Cell Transplantation, Medical Center, Faculty of Medicine, University of Freiburg, Freiburg, Germany

6 Department of Hematology and Oncology, Medical Center, University of Schleswig-Holstein, Campus Lübeck, Lübeck, Germany

7 Hematology Unit, IRCCS Istituto Tumori "Giovanni Paolo II", Bari, Italy

8 Oncology-Hematology Department, A.O. "Bianchi-MelacrinoMorelli", Reggio Calabria, Italy
Recently, ropeginterferon $\alpha-2 b$ was approved by European Medicinal Agency as first line for patients without symptomatic splenomegaly [3]. Ruxolitinib is second-line for patients who are refractory and/or intolerant to hydroxyurea [4]; other treatments include busulfan, pipobroman [5], and nonpegylated and pegylated interferons (off-label) [1, 6, 7], but use is limited by side effects and safety concerns. Additional, targeted therapies are therefore needed.

Up to $98 \%$ of patients with PV bear the $J A K 2^{\mathrm{V} 617 \mathrm{~F}}$ gene mutation, which activates erythropoietin receptor signaling pathways. Givinostat is a histone-deacetylase (HDAC) inhibitor that selectively targets $J A K 2^{\mathrm{V} 617 \mathrm{~F}}$ cell growth, reducing hematopoietic cell proliferation [8]. The efficacy and safety of givinostat alone or with hydroxyurea has previously been evaluated in two studies in $J A K 2^{\mathrm{V} 617 \mathrm{~F}}$

9 Department of Hematology Oncology, Charité' Medical School, Campus Benjamin Franklin, Berlin, Germany

10 U.O. Hematology, San Bortolo Hospital, Vicenza, Italy

11 Hematology and Stem Cells Transplantation Unit, Campus BioMedico, University Hospital, Rome, Italy

12 U.O. Clinical Hematology, Presidio Ospedaliero "Spirito Santo"A.S.L. Azienda Sanitaria Locale, Pescara, Italy

13 Centre for Medical Education, Queen's University Belfast, Belfast, UK

14 Service d'Oncologie Hématologie, Hospital Saint Vincent de Paul —GHICL Lille, Lille, France

15 Service d'Oncologie Hématologie Clinique, CHU AmiensHospital Sud, Amiens, France

16 Mayo Clinic Cancer Center, Scottsdale, AZ, USA

17 New York University School of Medicine \& Perlmutter Cancer Center/ NYU Langone Health, New York, NY, USA

18 Clinical R\&D Department, Italfarmaco S.p.A., Cinisello Balsamo, Italy 
positive PV $[9,10]$. Although these studies confirmed the positive risk-benefit of givinostat, they did not provide comprehensive efficacy evidence for givinostat monotherapy, and did not identify the most appropriate dose. The current study was therefore conducted to support givinostat monotherapy development in $\mathrm{PV}$, aiming to determine the maximum tolerated dose (MTD), and to assess safety and efficacy of this dose.

This multinational, open-label, nonrandomized study was conducted in two parts. Part A (Phase Ib) was dose escalation, with the first 4-week cycle determining the MTD. Part B (Phase II), the proof of concept phase, then evaluated efficacy and safety at this MTD. Full details of the methods are in the supplement. Both parts had 24-week treatment periods, with patients receiving six four-week cycles of givinostat. In Part A, since givinostat $50 \mathrm{mg}$ twice daily (BID) was previously well tolerated, the first cohort of three patients received $100 \mathrm{mg}$ BID, with the dose to be escalated by $50 \mathrm{mg}$ BID in each subsequent cohort according to a $3+3$ design, adopting a modified Fibonacci escalation scheme, although only after the third patient had been followed for a minimum of one cycle, and tolerability data had been evaluated by the Safety Review Team (Supplementary Table 1). For Part B, patients initially received givinostat at the MTD, with modification permitted to achieve an optimized dose, balancing tolerability, and response.

Eligible patients were aged $\geq 18$ years, with a confirmed $\mathrm{PV}$ diagnosis, $J A K 2^{\mathrm{V} 617 \mathrm{~F}}$ positivity assessed by centralized quantitative real-time polymerase chain reaction, and active/ not controlled disease, defined as: (1) hematocrit $\geq 45 \%$ or $<45 \%$ with phlebotomy, and (2) platelet count $>400 \times 10^{9} / 1$, and (3) white blood cell count $>10 \times 10^{9} / 1$. Main exclusion criteria were: absolute neutrophil count $<1.2 \times 10^{9} /$; prior $J A K 2$ or HDAC inhibitor treatment; systemic treatment for cMPNs other than aspirin; hydroxyurea, interferon alpha, or anagrelide within 28,14 , or 7 days before enrollment, respectively. All patients provided informed consent. Study registration: ClinicalTrials.gov (NCT01901432).

The primary objectives of Part A were to determine givinostat's MTD, and to characterize safety and tolerability in terms of treatment-related adverse events (AEs). Secondary endpoints were to evaluate overall response after three and six cycles (using the clinico-hematological European LeukemiaNet (ELN) response criteria [11]), and to characterize pharmacokinetics. For Part B, primary objectives were to evaluate overall response, safety and tolerability after three cycles. Secondary endpoints were to evaluate overall response, safety and tolerability after six cycles, and to characterize pharmacokinetics. Exploratory endpoints are in the supplement.

Twelve patients were studied in Part A, with 35 in Part B (Supplementary Fig. 1, Supplementary Tables 2 and 3). In
Part A, during the first cycle one patient receiving givinostat $100 \mathrm{mg}$ BID experienced dose-limiting toxicity: Grade 3 dyspepsia, drug related, resolving with sequelae after treatment. Three additional patients therefore received $100 \mathrm{mg}$ BID, none of whom had dose-limiting toxicity during Cycle 1. Although escalation to higher doses was permitted, the Safety Review Team agreed the MTD was $100 \mathrm{mg}$ BID, given: (a) thrombocytopenia is a known side effect of HDAC inhibitors; (b) a platelet count decrease was observed in subsequent cycles; (c) as givinostat is a chronic treatment, it was preferable to not expose patients to higher doses that could be poorly tolerated during chronic treatment. To more accurately define givinostat's MTD, three additional patients received an intermediate dose $(150 \mathrm{mg}$ daily). Finally, three patients received $50 \mathrm{mg}$ BID, to investigate safety, pharmacokinetics, and pharmacodynamics of this dose. A total of $66.7 \%$ of patients experienced at least one drug-related AE, mainly Grade 1 or 2 (Supplementary Table 4), most commonly thrombocytopenia (33.3\% of patients). Two patients (16.7\%) experienced a serious AE (thrombophlebitis and myocardial infarction), neither drug-related; no patient died. Two patients withdrew due to drug-related AEs (dyspepsia [Grade 3] and thrombocytopenia [Grade 4]), both with $100 \mathrm{mg}$ BID.

The overall response rate in Part A was above $70 \%$ (Fig. 1). One patient achieved complete response after three cycles, and one after six cycles. Median givinostat $T_{\max }$ was 1.5-4 h (Supplementary Table 5), with steady-state reached by day 28 of Cycle 1 (the first repeat-dose pharmacokinetic evaluation). After three cycles, givinostat normalized hematological parameters in $45.5-54.5 \%$ of patients (Supplementary Table 6), normalized spleen volume in $54.5 \%$, resolved disease-related symptoms in $63.6 \%$ (Supplementary Table 7), and reduced pruritus and JAK2-mutated allele burden (Supplementary Table 8).

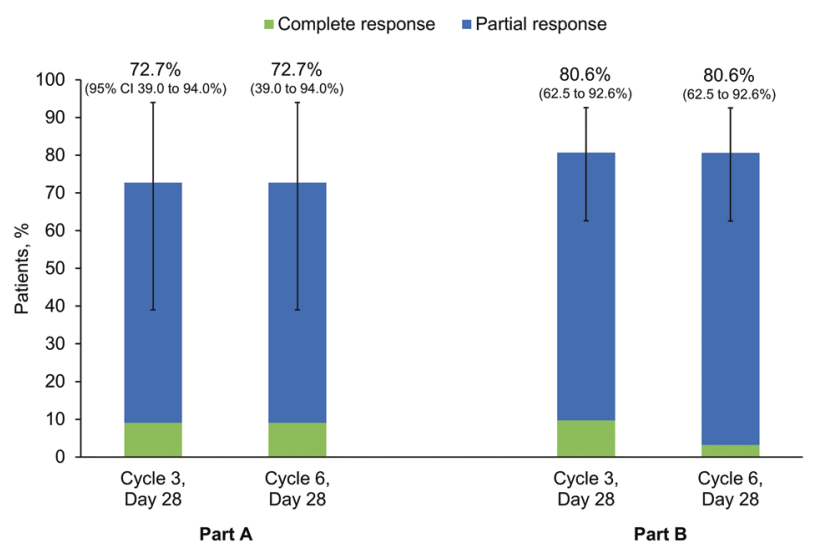

Fig. 1 Parts A and B: therapeutic response evaluation (intentionto-treat population). Data are from 11 patients in Part $\mathrm{A}$ and 31 patients in Part B. 
At the end of Cycles 3 and 6 of Part B, 80.6\% of patients were responders (Fig. 1), with three achieving complete response after three cycles and one after six. Overall, $94.3 \%$ of patients had at least one drug-related AE, the majority Grade 1 or 2 and none Grade 4 or 5 , with most occurring during the first three cycles of treatment (152 out of 190 events). The most common were diarrhea (51.4\% of patients), thrombocytopenia $(45.7 \%)$, and increased blood creatinine $(37.1 \%)$ (Table 1). Two patients experienced a serious $\mathrm{AE}$, both during the first three cycles, one study drug-related (Grade 3 diarrhea resolving in 7 days without therapy, with study drug temporarily discontinued). Three patients withdrew, one due to study drug-related AEs (Grade 3 neutropenia and Grade 2 thrombocytopenia, both resolving). The other two were withdrawn by their investigators (Supplementary Fig. 1). No patient died, and there were no clinically relevant vital signs or ECG values.

Overall, Part B pharmacokinetics was similar to Part A at comparable doses (Supplementary Tables 9 and 10). Improvements were seen in all individual response criteria (Supplementary Fig. 2; Supplementary Table 11), with white blood cell and platelet counts normalized in $90.3 \%$ and $74.2 \%$ of patients after three cycles, respectively, and hematocrit in $77.4 \%$ and $48.4 \%$ after three and six cycles, respectively. Improvements were observed in diseaserelated symptoms assessed by Myeloproliferative Neoplasm Symptom Assessment Form quality of life (QoL) questionnaire, especially during Cycle 6 (Supplementary Table 12), with a reduction in the proportion with severe pruritus (Score 7-10; Supplementary Fig. 3). Approximately 50\% had no headache (Supplementary Table 12), and no patients had severe headache (Supplementary Fig. 4). The proportion of patients without microvascular symptoms improved from baseline $(38.7 \%)$ to Cycle 6 (51.6\%; Supplementary Table 12), with a low proportion having severe symptoms (6.5-12.9\%; Supplementary Fig. 5). A total of $19.4 \%$ had a spleen volumetric index reduction of at least $35 \%$ during treatment, with total spleen normalization in two and three patients after three and six cycles, respectively, and a moderate reduction in $J A K 2^{\mathrm{V} 617 \mathrm{~F}}$ allele burden (Supplementary Table 12). Finally, differential gene expression was observed (Supplementary Fig. 6), with upregulation for GLRX, STAT4 and HDAC3, and downregulation for $M Y C$.

The study aims were achieved, with the MTD, $100 \mathrm{mg}$ BID, determined in Part A, and this dose effective in Part $B$. In addition to the high overall response rate, givinostat had a positive impact on individual clinico-hematological ELN criteria, both hematological parameters and diseaserelated symptoms. The three hematological parameters, all abnormal at study entry, were normalized in the majority of patients, and givinostat improved key disease-related
Table 1 Part B: patients with study drug-related treatment-emergent AEs, overall and by system organ class and preferred term (including only preferred terms reported by one or more patient with Grade 3 events) (safety population).

\begin{tabular}{|c|c|c|c|c|}
\hline \multirow[t]{2}{*}{ System organ class preferred term } & \multicolumn{2}{|c|}{ Grade 3} & \multicolumn{2}{|c|}{$\begin{array}{l}\text { Any } \\
\text { grade }\end{array}$} \\
\hline & $N$ & $\%$ & $N$ & $\%$ \\
\hline Patients with any drug-related $\mathrm{AE}$ & 10 & 28.6 & 33 & 94.3 \\
\hline Blood and lymphatic system disorders & 3 & 8.6 & 18 & 51.4 \\
\hline Anemia & 2 & 5.7 & 6 & 17.1 \\
\hline Neutropenia & 1 & 2.9 & 2 & 5.7 \\
\hline Thrombocytopenia & 1 & 2.9 & 16 & 45.7 \\
\hline Cardiac disorders & $0^{\mathrm{a}}$ & 0 & 1 & 2.9 \\
\hline Gastrointestinal disorders & 4 & 11.4 & 26 & 74.3 \\
\hline Diarrhea & 4 & 11.4 & 18 & 51.4 \\
\hline $\begin{array}{l}\text { General disorders and administration site } \\
\text { conditions }\end{array}$ & 2 & 5.7 & 9 & 25.7 \\
\hline Asthenia & 2 & 5.7 & 8 & 22.9 \\
\hline Investigations & $0^{\mathrm{a}}$ & 0 & 19 & 54.3 \\
\hline Metabolism and nutrition disorders & 1 & 2.9 & 8 & 22.9 \\
\hline Hypocalcemia & 1 & 2.9 & 4 & 11.4 \\
\hline Nervous system disorders & $0^{\mathrm{a}}$ & 0 & 5 & 14.3 \\
\hline Renal and urinary disorders & $0^{\mathrm{a}}$ & 0 & 2 & 5.7 \\
\hline Respiratory, thoracic and mediastinal disorders & $0^{\mathrm{a}}$ & 0 & 1 & 2.9 \\
\hline Skin and subcutaneous tissue disorders & 1 & 2.9 & 6 & 17.1 \\
\hline Rash & 1 & 2.9 & 1 & 2.9 \\
\hline
\end{tabular}

Data are from 35 patients. Grades are based on the National Cancer Institute Common Terminology Criteria for Adverse Events Version 4.03, where Grade 1 are mild events, Grade 2 are moderate, Grade 3 are severe, Grade 4 are life-threatening, and Grade 5 events result in death. There were no Grade 4 or 5 events in Part B of the study.

$A E$ adverse event.

${ }^{\text {a }}$ There were no Grade 3 events for these system organ classes.

symptoms, notably pruritus with complete resolution in many patients, with an associated positive impact on QoL. A reduction in JAK2-mutated allele burden was observed in both parts of the study, and Part B provided clear evidence of differential gene expression with givinostat, consistent with disease pathway regulation. Overall, givinostat was well tolerated with no new safety concerns. Unlike previous studies, the recruited population had active or not controlled disease, and were both high- and low-risk, making comparisons difficult. However, the observed response was greater than for other PV therapies [12-15]. For example, in a study comparing interferon to hydroxyurea, $45 \%$ of patients had a hematologic response to either therapy [12], whereas in a second study, $40 \%$ of patients had a response of any type to ruxolitinib [13], and in a third the overall response to the HDAC inhibitor vorinostat was $35 \%$, with significant side effects resulting in a high rate of study withdrawal [15]. 
In conclusion, these data support givinostat monotherapy development in the defined PV target population.

Acknowledgements This study was funded by Italfarmaco S.p.A. David Young of Young Medical Communications and Consulting Ltd, a medical writer supported by funding from Italfarmaco S.p.A., provided drafts and editorial assistance to the authors during preparation of this paper. The authors would like to thank the investigators who recruited patients, including Dr Andrzej Hellmann, and the patients at the investigative sites for their support of this study.

\section{Compliance with ethical standards}

Conflict of interest AR has received honoraria for consultancy, and travel support from Italfarmaco, Gilead, Amgen, Novartis, Pfizer, Celgene, Sanofi, Astellas and Roche. AI has received speaker honoraria from Novartis, Pfizer and Incyte. AMV has received honoraria for advisory board participation from Novartis, Celgene, Incyte, CTI and Italfarmaco, and for lectures from Novartis, and CTI. N.v.B. received research funding from Novartis. MFM has received honoraria and has attended advisory boards with Novartis, and has received honoraria from Celgene. RAM has received honoraria as a consultant to Novartis, Sierra Oncology, and La Jolla Oncology, and research support from Incyte, Genentech, Celgene, CTI, and Abbvie. RT has advised and received honoraria from Italfarmaco. $\mathrm{PB}, \mathrm{SM}$, and SDT are employees of Italfarmaco SpA, the sponsor of the study. RN, AG, BM, AP, GC, MDM, SL, NC, J-PM, APancrazzi, FG have no conflicts to disclose.

Publisher's note Springer Nature remains neutral with regard to jurisdictional claims in published maps and institutional affiliations.

Open Access This article is licensed under a Creative Commons Attribution 4.0 International License, which permits use, sharing, adaptation, distribution and reproduction in any medium or format, as long as you give appropriate credit to the original author(s) and the source, provide a link to the Creative Commons license, and indicate if changes were made. The images or other third party material in this article are included in the article's Creative Commons license, unless indicated otherwise in a credit line to the material. If material is not included in the article's Creative Commons license and your intended use is not permitted by statutory regulation or exceeds the permitted use, you will need to obtain permission directly from the copyright holder. To view a copy of this license, visit http://creativecommons. org/licenses/by/4.0/.

\section{References}

1. McMullin MF, Harrison CN, Ali S, Cargo C, Chen F, Ewing J, et al. A guideline for the diagnosis and management of polycythaemia vera. A British Society for Haematology Guideline. Br J Haematol. 2019;184:176-91.

2. Demuynck T, Verhoef G, Delforge M, Vandenberghe P, Devos T. Polycythemia vera and hydroxyurea resistance/intolerance: a monocentric retrospective analysis. Ann Hematol. 2019;98:1421-6.

3. Kiladjian J-J, Cassinat B, Soret-Dulphy J, Verger E, Roy L, Rey J, et al. Molecular response to hydroxyurea and ropeginterferon alfa2B in the PROUD-PV randomized Phase 3 trial. Haematologica. 2017;102:S787.

4. Mesa RA. New guidelines from the NCCN for polycythemia vera. Clin Adv Hematol Oncol. 2017;15:848-50.

5. Kiladjian J-J, Gardin C, Renoux M, Bruno F, Bernard J-F. Longterm outcomes of polycythemia vera patients treated with pipobroman as initial therapy. Hematol J. 2003;4:198-207.

6. Kiladjian J-J, Chomienne C, Fenaux P. Interferon-alpha therapy in bcr-abl-negative myeloproliferative neoplasms. Leukemia. 2008;22:1990-8.

7. Yacoub A, Mascarenhas J, Kosiorek H, Prchal JT, Berenzon D, Baer MR, et al. Pegylated interferon alfa-2a for polycythemia vera or essential thrombocythemia resistant or intolerant to hydroxyurea. Blood. 2019;134:1498-509.

8. Guerini V, Barbui V, Spinelli O, Salvi A, Dellacasa C, Carobbio A, et al. The histone deacetylase inhibitor ITF2357 selectively targets cells bearing mutated JAK2V617F. Leukemia. 2008;22:740-7.

9. Rambaldi A, Dellacasa CM, Finazzi G, Carobbio A, Ferrari ML, Guglielmelli P, et al. A pilot study of the histone-deacetylase inhibitor givinostat in patients with JAK2V617F positive chronic myeloproliferative neoplasms. Br J Haematol. 2010;150:446-55.

10. Finazzi G, Vannucchi AM, Martinelli V, Ruggeri M, Nobile F, Specchia G, et al. A phase II study of givinostat in combination with hydroxycarbamide in patients with polycythaemia vera unresponsive to hydroxycarbamide monotherapy. Br J Haematol. 2013;161:688-94.

11. Barosi G, Rosti V. Novel strategies for patients with chronic myeloproliferative disorders. Curr Opin Hematol. 2009;16:129-34.

12. Gisslinger H, Klade C, Georgiev P, Skotnicki A, GerchevaKyuchukova L, Egyed M, et al. Final results from PROUD-PV a randomized controlled Phase 3 trial comparing ropeginterferon alfa- $2 b$ to hydroxyurea in polycythemia vera patients. Blood. 2016;128:475.

13. Pugliese N, Giordano C, Nappi D, Luciano L, Cerchione C, Annunziata $\mathrm{M}$, et al. Adding hydroxyurea in combination with ruxolitinib improves clinical responses in hyperproliferative forms of myelofibrosis. Cancer Med. 2019;8:2802-9.

14. Kiladjian J-J, Guglielmelli P, Griesshammer M, Saydam G, Masszi T, Durrant S, et al. Efficacy and safety of ruxolitinib after and versus interferon use in the RESPONSE studies. Ann Hematol. 2018;97:617-27.

15. Andersen CL, McMullin MF, Ejerblad E, Zweegman S, Harrison C, Fernandes S, et al. A phase II study of vorinostat (MK-0683) in patients with polycythaemia vera and essential thrombocythaemia. Br J Haematol. 2013;162:498-508. 